\title{
Reversible beam heater for suppression of microbunching instability by transverse gradient undulators
}

\author{
Tao Liu, ${ }^{1,2,3}$ Weilun Qin, ${ }^{2,4}$ Dong Wang, ${ }^{3}$ and Zhirong Huang, ${ }^{2, *}$ \\ ${ }^{1}$ University of Chinese Academy of Sciences, Beijing 100049, China \\ ${ }^{2}$ SLAC National Accelerator Laboratory, Menlo Park, Califonia 94025, USA \\ ${ }^{3}$ Shanghai Institute of Applied Physics, Chinese Academy of Sciences, Shanghai 201800, China \\ ${ }^{4}$ Institute of Heavy Ion Physics, School of Physics, Peking University, Beijing 100871, China
}

(Received 31 May 2017; published 2 August 2017)

\begin{abstract}
The microbunching instability driven by beam collective effects in a linear accelerator of a free-electron laser (FEL) facility significantly degrades the electron beam quality and FEL performance. A conventional method to suppress this instability is to introduce an additional uncorrelated energy spread by laser-electron interaction, which has been successfully operated in the Linac Coherent Light Source and Fermi@Elettra, etc. Some other ideas are recently proposed to suppress the instability without increasing energy spread, which could benefit the seeded FEL schemes. In this paper, we propose a reversible electron beam heater using two transverse gradient undulators to suppress the microbunching instability. This scheme introduces both an energy spread increase and a transverse-to-longitudinal phase space coupling, which suppress the microbunching instabilities driven by both longitudinal space charge and coherent synchrotron radiation before and within the system. Finally the induced energy spread increase and emittance growth are reversed. Theoretical analysis and numerical simulations are presented to verify the feasibility of the scheme and indicate the capability to improve the seeded FEL radiation performance.
\end{abstract}

DOI: 10.1103/PhysRevAccelBeams.20.082801

\section{INTRODUCTION}

An x-ray free electron laser (FEL) is able to provide ultrashort, extremely bright radiation for advanced applications in biology, chemistry and material science at the ultrafast time and atomic scale [1]. In recent years, it has been successful with the operation of the FEL facilities in soft and hard x-ray regimes [2-5]. A high current electron beam is achievable by magnetic compression in a linear accelerator, while in the meantime the beam quality is deteriorated due to the microbunching instability driven by relevant collective effects such as longitudinal space charge (LSC) [6] and coherent synchrontron radiation (CSR) [7-11], which arises from the small energy and density perturbation of the initial beam and is accumulated during acceleration, compression and transport. Afterwards the amplified instability would significantly degrade the FEL performance.

The microbunching instability can be suppressed by manipulating the phase space of the electron beam. A conventional method which has been successfully implemented in linear accelerators [12-14], is using a laser heater system to increase the uncorrelated energy spread of

\footnotetext{
*zrh@slac.stanford.edu
}

Published by the American Physical Society under the terms of the Creative Commons Attribution 4.0 International license. Further distribution of this work must maintain attribution to the author(s) and the published article's title, journal citation, and DOI. the beam. The laser heater suppresses the microbunching instability at the price of an enlarged slice energy spread. This is typically tolerable for operation of self-amplified spontaneous emissions (SASE) FELs. However, for an advanced $x$-ray FEL using external seed laser, the FEL gain is limited to some degree [15] and a smaller uncorrelated energy spread is desirable.

In recent years, alternative methods based on the reversible heating concept (i.e., increased energy spread would be reversed) have been proposed, such as using $\mathrm{rf}$ transverse deflecting structures, bending magnets, or transverse gradient undulators [16-19]. The reversible heater based on transverse deflecting structures (TDSs) [16] was proposed to suppress the microbunching instabilities driven by both LSC and CSR without degrading the beam quality. The drawback of this scheme is the extremely tight energyjitter tolerance. For the scheme involving bending magnets [17], it suppresses the microbunching amplification within the system due to the transverse-to-longitudinal coupling, but the initial modulation cannot be suppressed, and it leads to an inconvenient deflection of the downstream beam line. For the schemes involving transverse gradient undulators (TGUs), the original scheme [18] suppresses the microbunching instabilities driven by both LSC and CSR before and within the system, but it leads to an energy spread increase and a transverse emittance dilution. The second scheme based on TGUs [19] overcomes the disadvantage of the former one, but it reverses all of the coupling terms, thus such a system cannot suppress the LSC driven microbunching instability before the system (i.e. the initial 
modulation). In addition, an equivalent reversible system replacing TDSs with bending magnets was proposed in Ref. [20]. The scheme creates the same overall transport matrix as the one using the TDS system, but it still has a beam line deflection and the full beam dynamics issues are not explored.

In this paper, we expand the idea of the reversible heater without TDSs [20] and propose a method of a reversible heater based on TGUs. Previously, the transport matrix and beam dynamics of a TGU has been investigated preliminarily [18], which plays a role of a bending magnet to provide a required dispersion. We present a more accurate transport theory within a TGU which impacts on the overall beam optics. In this scheme, we induce an uncorrelated energy spread and a transverse-to-longitudinal coupling to suppress both LSC and CSR driven microbunching instabilities before and within the system, and finally reverse the energy spread heating and emittance dilution at the end of the system. In Sec. II, we first propose our method and describe the layout of the overall scheme briefly. Then we discuss the beam dynamics of the TGU, derive the overall matrix and describe the theory of the reversibility for the proposed system. Through the system the process from heating to reversion of the slice energy spread is presented, as well as the transverse emittance preservation. Next we discuss the suppression of microbunching instabilities driven by both LSC and CSR before and within the system. In addition, nonlinear effects and wakefield working on the six-dimensional phase space are also included here. In Sec. III, we present numerical simulation results for the demonstration of the scheme. Besides, some practical issues like jitter and tolerance are considered in Sec. IV. A conclusion is drawn in Sec. V.

\section{METHODS}

In this section, we consider a linear accelerator employing a single stage compressor for a soft x-ray FEL to simplify the theoretical analysis. The proposed layout of the reversible heating system is shown in Fig. 1. We use an initial Gaussian beam without any correlation in the study. For the two rf linacs, the first linac (L1) provides a time-energy chirp before the first TGU (T1). While the second linac (L2) not only provides the additional chirp for bunch compression, but also leaks out a coupling term downstream the second TGU (T2).
For the two TGUs (T1\&T2), T1 induces a dispersion for energy spread heating and transverse-to-longitudinal mixing, while T2 after the bunch compressor is added to remove the increased energy spread and transverse emittance. In addition, quadrupoles, harmonic cavities, and one sextupole are adopted for beam optics up to the second order, which makes sure both energy spread and emittance are reversed and an additional transverse-to-longitudinal coupling is leaked out for microbunching suppression. It is worth noting that the scheme is also appropriate for an initially chirped beam due to the use of L1. In the following subsections, we will elaborate the operating principle.

\section{A. Beam dynamics of a TGU}

A TGU with a transverse magnetic field variation was initially proposed to improve FEL performance of an electron beam with a large energy spread [21,22] and being tested for FEL driven by laser plasma accelerator [23]. In this study, we make use of the dispersion generated by a TGU. Instead of a dipole, the motion in a TGU associated with a correcting coil relies on a longitudinalalternating and transverse-dependent magnetic field that does not cause a deflection of the downstream beam line but still produces a transverse dispersion. By means of the magnetic field in a TGU [24], we derive the motion equation expressed by the first order transport matrix [25]

$$
R_{T}=\left(\begin{array}{ccc}
\cos \left(k_{x} z\right) & \frac{1}{k_{x}} \sin \left(k_{x} z\right) & 0 \\
-k_{x} \sin \left(k_{x} z\right) & \cos \left(k_{x} z\right) & 0 \\
0 & 0 & \cos \left(k_{y} z\right) \\
0 & 0 & -k_{y} \sin \left(k_{y} z\right) \\
-\eta_{c} k_{x} \sin \left(k_{x} z\right) & -\eta_{c}\left(1-\cos \left(k_{x} z\right)\right) & 0 \\
0 & 0 & 0 \\
0 & 0 & \eta_{c}\left(1-\cos \left(k_{x} z\right)\right) \\
0 & 0 & \eta_{c} k_{x} \sin \left(k_{x} z\right) \\
\frac{1}{k_{y}} \sin \left(k_{y} z\right) & 0 & 0 \\
\cos \left(k_{y} z\right) & 0 & 0 \\
0 & 1 & k_{x}^{2} \eta_{c} \eta_{\alpha} z-\eta_{c}^{2} k_{x}\left(k_{x} z-\sin \left(k_{x} z\right)\right) \\
0 & 0 & 1
\end{array}\right),
$$

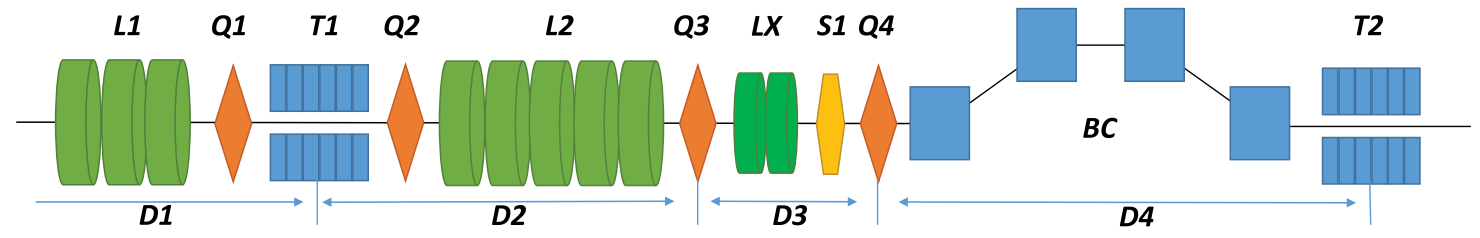

FIG. 1. Layout of the proposed reversible beam heater based on TGUs. The first TGU (T1) is located between the first and the second rf linacs (L1, L2) and the second one (T2) is located downstream of the bunch compressor (BC). The layout also includes several quadrupoles (Q1-Q4), a harmonic cavity (LX) and a sextupole (S1). According to Eq. (6), D1—D4 indicate the relative lengths where Q1 and Q2 are switched off. 
where the focusing parameters are $k_{x}^{2}=\alpha K_{0}^{2} / 2 \gamma_{0}^{2} \eta_{c}$ and $k_{y}^{2}=K_{0}^{2}\left(k_{u}^{2}+\alpha^{2}+\alpha \alpha_{c}\right) / 2 \gamma_{0}^{2} . \alpha, K_{0}$, and $z$ are the transverse gradient of the magnetic field, the dimensionless undulator parameter and the effective undulator length of a TGU, respectively. $\alpha_{c}$ is the magnetic gradient of the correcting coil with the same unit of $\alpha . \eta_{\alpha}$ is the resonant dispersion of a TGU defined as $\eta_{\alpha}=\left(2+K_{0}^{2}\right) / \alpha K_{0}^{2}$ and $\eta_{c}$ is an equivalent dispersion defined as $\eta_{c}=1 /\left(\alpha-\alpha_{c}\right)$. Typically, under the condition of $\alpha=\alpha_{c}$, the horizontal focusing is removed and the TGU matrix can be approximated as the simplified formula used in Ref. [18], except for the vertical focusing and a modified factor in terms of $R_{56}$. In our system we adopt the simplified formula for theoretical analysis and the accurate formula in Eq. (1) for beam optics and simulations, in which we define the TGU strength $\theta=R_{26}$.

\section{B. Overall matrix}

Starting from a simple case consisting of a rf linac, a TGU and a bunch compressor (L1, T1 \& BC) [18], both the initial and the internal collective effects driven microbunching instabilities are suppressed, while at the end the slice energy spread is increased and the projected emittance is also diluted. By adding the second TGU (T2) [19], these degrading effects can be removed, however the microbunching instability driven by the initial energy modulation would be amplified rather than suppressed. Hence, the adoption of the second rf linac (L2) combining with quadrupoles can solve the problem that reverses the increased energy spread and the diluted emittance, meanwhile it also leaks out the transverse-to-longitudinal coupling term to suppress the effects of the initial modulation.

In order to facilitate the theoretical analysis of the scheme in Fig. 1, we consider here the four-dimensional linear transport matrices acting on the phase space vector $\left(x, x^{\prime}, s, \delta\right)$. Following Ref. [20], we disregard the transport length, quadrupoles, sextupoles, and harmonic cavities. As a consequence, the overall suppression system from the entrance of the first linac to the exit of the second TGU module can be given by

$$
R=R_{T}\left(\theta_{2}\right) R_{B C}\left(R_{56}\right) R_{L}\left(h_{2}\right) R_{T}\left(\theta_{1}\right) R_{L}\left(h_{1}\right),
$$

where the subscript of $R$ matrix and the parameter in the bracket are the type and the strength of each element, respectively. Under the symplectic condition and the thinlens approximation, the matrix $R$ is expressed as

$R=\left(\begin{array}{cccc}1 & 0 & 0 & 0 \\ -h_{2} \theta_{1} \theta_{2} & 1 & h_{2} \theta_{2}+h_{1}\left(\theta_{1}+\theta_{2}\right) & \theta_{1}+\theta_{2} \\ -\left(1+h_{2} R_{56}\right) \theta_{1}-\theta_{2} & 0 & 1+\left(h_{1}+h_{2}\right) R_{56} & R_{56} \\ -h_{2} \theta_{1} & 0 & h_{1}+h_{2} & 1\end{array}\right)$.
As mentioned above, the requirements of the matrix agreed with the design are to remove $R_{25}$ and leak out $R_{26}$ in Eq. (3). As a result, the specified condition is

$$
\theta_{2}=-\frac{h_{1} \theta_{1}}{h_{1}+h_{2}}
$$

and the matrix becomes

$$
R=\left(\begin{array}{cccc}
1 & 0 & 0 & 0 \\
\frac{h_{1} h_{2} \theta_{1}^{2}}{h_{1}+h_{2}} & 1 & 0 & \frac{h_{2} \theta_{1}}{h_{1}+h_{2}} \\
-\frac{h_{2} \theta_{1}}{h_{1}+h_{2}} \frac{1}{C} & 0 & \frac{1}{C} & R_{56} \\
-h_{2} \theta_{1} & 0 & h_{1}+h_{2} & 1
\end{array}\right)
$$

with a compression factor $C=1 /\left[1+\left(h_{1}+h_{2}\right) R_{56}\right]$.

A practical case requires a full beam optics each element included, therefore we need to do the point-to-point imaging from the middle of $\mathrm{T} 1$ to the middle of $\mathrm{T} 2$ by inserting quadrupoles to work out a complete reversible system. For instance, considering quadrupoles Q3, Q4 and the length of each element, we can propose the practical system characterized by the effective matrix

$$
\begin{aligned}
R= & R_{T}\left(\theta_{2}\right) R_{B C}\left(R_{56}\right) R_{D}\left(D_{4}\right) R_{Q}\left(K_{4}\right) R_{D}\left(D_{3}\right) \\
& \cdot R_{Q}\left(K_{3}\right) R_{D}\left(D_{2}\right) R_{L}\left(h_{2}\right) R_{T}\left(\theta_{1}\right) R_{L}\left(h_{1}\right),
\end{aligned}
$$

where $R_{D}$ and $R_{Q}$ represent the matrices of drift space and quadrupole, respectively. Particularly, all the lengths of the elements and the separation distances are extracted individually and recombined into the drift matrices. For instance, the length $D_{2}$ means the distance from the middle of the first TGU to the middle of the downstream quadrupole. Note that we removed two drift matrices for simplify, including the first one from the initial to the middle of the first TGU and the final one from the middle of the second TGU to the end, which do not impact on the essence of the case. Thus the condition specified in Eq. (4) is changed to

$$
\begin{aligned}
& K_{3}=\frac{D_{4}\left(h_{1}+h_{2}\right) \theta_{2}-\left(D_{2}+D_{3}\right) h_{1} \theta_{1}}{D_{2} D_{3} h_{1} \theta_{1}}, \\
& K_{4}=\frac{-\left(D_{3}+D_{4}\right)\left(h_{1}+h_{2}\right) \theta_{2}+D_{2} h_{1} \theta_{1}}{D_{3} D_{4}\left(h_{1}+h_{2}\right) \theta_{1}},
\end{aligned}
$$

which indicates that $\theta_{2}$ is independent of $h_{1}, h_{2}$, and $\theta_{1}$ when quadrupoles are included, and we can adjust quadrupoles other than TGU to satisfy the requirements of the system. The overall matrix is given by 
$R=\left(\begin{array}{cccc}-\frac{h_{1} \theta_{1}}{\left(h_{1}+h_{2}\right) \theta_{2}} & 0 & 0 & 0 \\ R_{21} & -\frac{\left(h_{1}+h_{2}\right) \theta_{2}}{h_{1} \theta_{1}} & 0 & \frac{h_{2} \theta_{2}}{h_{1}} \\ -\frac{h_{2} \theta_{1}}{h_{1}+h_{2}} \frac{1}{C} & 0 & \frac{1}{C} & R_{56} \\ -h_{2} \theta_{1} & 0 & h_{1}+h_{2} & 1\end{array}\right)$

with the term $R_{21}$ as

$$
\begin{aligned}
R_{21}= & h_{1} D_{3}\left(h_{1}+h_{2}\right) \theta_{1} \theta_{2}-\left(h_{1}+h_{2}\right)^{2} D_{4} \theta_{2}^{2} \\
& -h_{1}^{2} D_{2} \theta_{1}^{2}-h_{1} h_{2} D_{2} D_{4}\left(h_{1}+h_{2}\right) \theta_{1}^{2} \theta_{2}^{2},
\end{aligned}
$$

which has a similar form as in Eq. (5). The residual term $R_{51}$ represents the suppression factor for the initial modulation driven microbunching instability.

\section{Reversible heating and emittance preservation}

In the reversible heating system, a transverse-tolongitudinal coupling and an additional slice energy spread are imposed to the initial beam. To view the slice energy spread change, we adopt the similar method of tracking beam in matrix as Ref. [18] to calculate the evolution of the slice energy spread in the process. An initial beam with Gaussian distribution can be assumed as

$f\left(x_{0}, x_{0}^{\prime}, s_{0}, \delta_{0}\right)=A_{0} \exp \left(-\frac{x_{0}^{2}}{2 \sigma_{x_{0}}^{2}}-\frac{x_{0}^{\prime 2}}{2 \sigma_{x_{0}^{\prime}}^{2}}-\frac{s_{0}^{2}}{2 \sigma_{s_{0}}^{2}}-\frac{\delta_{0}^{2}}{2 \sigma_{\delta_{0}}^{2}}\right)$.

For approximate treatment, we mark the order of magnitude of the beam parameters: beam size $\sigma_{x_{0}} \sim 10^{-4} \mathrm{~m}$, beam divergence $\sigma_{x_{0}^{\prime}} \sim 10^{-5} \mathrm{rad}$, bunch length $\sigma_{s_{0}} \sim 10^{-3} \mathrm{~m}$ and relative energy spread $\sigma_{\delta_{0}} \sim 10^{-5}$. Passing through the first two elements, i.e., energy chirp $h_{1}$ and TGU $\theta_{1}$, the beam distribution $f_{1}\left(x, x^{\prime}, s, \delta\right)$ would be obtained. By integrating the distribution function over $x, x^{\prime}$ and selecting a thin slice, e.g. $s=0$, we can obtain the slice distribution with respect to $\delta$ given by

$$
\begin{aligned}
& f_{1}(s=0, \delta) \\
& \quad=A_{1} \cdot \exp \left[-\frac{\delta^{2}\left(\theta_{1}^{2} \sigma_{x_{0}}^{2}+\sigma_{s_{0}}^{2}\right)}{2\left[h_{1}^{2} \theta_{1}^{2} \sigma_{x_{0}}^{2} \sigma_{s_{0}}^{2}+\sigma_{\delta_{0}}^{2}\left(\theta_{1}^{2} \sigma_{x_{0}}^{2}+\sigma_{s_{0}}^{2}\right)\right]}\right] .
\end{aligned}
$$

Due to $\sigma_{s_{0}} \gg \theta_{1} \sigma_{x_{0}}$, the slice energy spread can be approximately expressed as

$$
\sigma_{\delta}\left(z \rightarrow T_{1}\right) \approx \sqrt{\sigma_{\delta_{0}}^{2}+h_{1}^{2} \theta_{1}^{2} \sigma_{x_{0}}^{2}} \approx h_{1} \theta_{1} \sigma_{x_{0}},
$$

which is the so-called heated energy spread. Note that for the practical case of Eq. (6) under the condition of Eq. (7), the heated energy spread would evolve into $\sigma_{\delta}=$ $\left(h_{1}+h_{2}\right) \theta_{2} \sigma_{x_{0}}$ due to the quadrupoles affecting the dispersion before the bunch compressor. It would contribute to the suppression of the microbunching induced by CSR inside the bunch compressor. Following Eq. (12) without quadrupoles, the slice energy spread after the bunch compressor would be amplified by a compression factor written as

$$
\sigma_{\delta}(z \rightarrow B C) \approx \sqrt{\frac{\sigma_{\delta_{0}}^{2}+h_{1}^{2} \theta_{1}^{2} \sigma_{x_{0}}^{2}}{1+\left(h_{1}+h_{2}\right) R_{56}}} \approx C h_{1} \theta_{1} \sigma_{x_{0}} .
$$

While the quadrupoles are considered, the corresponding result is $\sigma_{\delta}=C\left(h_{1}+h_{2}\right) \theta_{2} \sigma_{x_{0}}$. Then passing through the overall system, the heated energy spread will be reversed by the second TGU given by

$$
\sigma_{\delta}\left(z \rightarrow T_{2}\right) \approx \sqrt{\frac{\sigma_{\delta_{0}}^{2}}{1+\left(h_{1}+h_{2}\right) R_{56}}}=C \sigma_{\delta_{0}}
$$

which is only related to the initial slice energy spread and the compression factor instead of the transverse beam size eventually.

Inevitably, the horizontal emittance would be diluted by the transverse-to-longitudinal coupling. We can derive the horizontal emittance evolution of an initial Gaussian beam along the beam line based on the transport matrix up to the second order, which is given by

$$
\begin{aligned}
\epsilon_{x}^{2}= & \sum_{i<j}\left(\sigma_{i}^{2} \sigma_{j}^{2}\left(R_{1 i} R_{2 j}-R_{1 j} R_{2 i}\right)^{2}\right)+\sum_{k, m<n}(4 R T(k m n)) \\
& +\sum_{k, m=n}(R T(k m n))+\sum_{p<q, u<v}(16 T T(p q u v)) \\
& +\sum_{p=q, u<v}(4 T T(p q u v))+\sum_{p=q, u=v}(T T(p q u v))
\end{aligned}
$$

with the abbreviations

$$
\begin{aligned}
R T(k m n) & =\sigma_{k}^{2} \sigma_{m}^{2} \sigma_{n}^{2}\left(R_{1 k} T_{2 m n}-R_{2 k} T_{1 m n}\right)^{2} \\
T T(p q u v) & =\sigma_{p}^{2} \sigma_{q}^{2} \sigma_{u}^{2} \sigma_{v}^{2}\left(T_{1 p q} T_{2 u v}-R_{2 p q} T_{1 u v}\right)^{2},
\end{aligned}
$$

where $R_{i j}$ and $T_{k m n}$ are the elements of the first- and second-order transport matrices with the subscript $i$ of each dimension, and $\sigma_{i}$ indicates the rms value of the beam in the $i$ th dimension. Based on Eq. (15) and the beam parameters given above, the bunch length is the most significant contribution factor to emittance. Without the second TGU, the terms $R_{15}$ and $R_{25}$ will be leaked out, leading to a serious growth of the horizontal emittance. With the proposed reversible system, the contribution from the bunch length is canceled according to Eq. (8) under the linear transport approximation, in which it only leaks out the term $R_{26}$ working on the initial small energy spread and leading to a small emittance growth by a few percent. 


\section{Suppression of microbunching instabilities}

To analyze the suppression effects, we consider the microbunching generated by two parts: the initial collective effects before the system and the internal collective effects inside the system.

The initial collective effects include the earlier LSC impedance and compressing stage before our suppression system, which lead to an original accumulation of the energy and density modulation. Typically in our case, the energy modulation induced by LSC in the first linac can be treated as an initial collective effect. The internal collective effects include the LSC and CSR effects inside out system. As we know, an initial energy modulation mainly contributes to the microbunching passing through bunch compressor directly, while an initial density modulation is usually converted into energy modulation by the internal collective effects before. Consequently, we can use the initial energy modulation and density modulation to indicate the initial modulation driven and the internal collective effects driven microbunching instabilities, respectively.

For the microbunching instability driven by an initial energy modulation, take into account an electron beam with an initial bunching factor $b_{0}\left(k_{0}\right)$ and a relative energy modulation $\delta_{m}\left(z_{0}\right)$. By transporting the beam through the overall system based on Eq. (8), we can obtain the final bunching factor without the internal collective effects which is given by

$$
\begin{aligned}
b_{f}\left(k_{f}\right)= & {\left[b_{0}\left(k_{0}\right)-i k_{f} R_{56} \delta_{m}\left(k_{f}\right)\right] \exp \left(-\frac{k_{f}^{2} R_{56}^{2} \sigma_{\delta_{0}}^{2}}{2}\right) } \\
& \times \exp \left(-\frac{k_{0}^{2} h_{2}^{2} \theta_{1}^{2} \sigma_{x_{0}}^{2}}{2\left(h_{1}+h_{2}\right)^{2}}\right)
\end{aligned}
$$

where the exponential term related to beam size plays an important role on suppressing microbunching besides the term related to compression. In square brackets, there are the initial microbunching and the accumulation from the initial energy modulation to the density modulation, in which typically, we have the relation of $\left|k_{f} R_{56} \delta_{m}\left(k_{f}\right)\right| \gg$ $\left|b_{0}\left(k_{0}\right)\right|$, and the component contributing to the microbunching instability is dominated by the initial energy modulation. Therefore the suppression factor related to the beam size should be the level that enables suppressing the microbunching instability due to the initial energy modulation.

On the other hand, we analyze the internal collective effects driven microbunching instability from an initial density modulation case. The analytical treatment follows Refs. $[9,17]$ and we briefly elaborate the fundamental process here. At the downstream of the first TGU, the electron beam is heated in the longitudinal phase space, where a coupling from the transverse phase space and an additional slice energy spread are generated. The $s-x$ coupling smears out the longitudinal density distribution, resulting in the suppression of energy modulation due to the internal LSC and CSR effects. In the bunch compressor, the increased energy spread plays a part in suppressing the conversion from energy modulation to density modulation, which suppresses CSR driven microbunching instability. It is noted that besides the initial density modulation, the initial energy modulation would also be amplified by the internal CSR effects in the bunching compressor, which is also suppressed by the increased energy spread. As a consequence, it suppresses the positive feedback amplification between energy modulation and density modulation, and the two modulations are hardly accumulated to the final bunching factor.

As described above, no matter energy modulation or density modulation, the induced microbunching instabilities would be suppressed by the reversible heating system. That indicates this system enables suppressing the microbunching instabilities driven by both the initial collective effects before the system and the internal collective effects inside the system.

\section{E. High order and wakefield effects}

It is worth noting that the high order effects and wakefield effects would deteriorate the reversible system [26]. According to Eq. (15), the nonlinear terms will contribute to the emittance dilution, particularly the terms related to the longitudinal position. Typically, the nonlinear energy chirp generated by the first linac or the initial beam will be converted to a nonlinear transverse distribution as a function of the longitudinal position after the first TGU written as $T_{255}=R_{26} T_{655}$. Besides, the nonlinear components of TGUs would not be ignored and the transport matrix should be extended to high order. To take into account the longitudinal wakefield of the linac and the CSR wake, the transverse phase space of the beam coupled with both $s$ and $\delta$ will be deteriorated, leading to a considerable increase by more than a factor of 10 . Furthermore, these effects also affect the energy chirp and the compression factor.

It is critical to remove the nonlinear effects on phase space, restore the energy spread and emittance, etc., in which wakefield effects also can be attributed to high order effects. Using the harmonic rf cavity not only keeps a large compression ratio, but also corrects the nonlinear transverse-to-longitudinal coupling to a higher level. In view of the complex coupling of the beam in our case, quadrupole also plays a role of the high order correction expressed as $T_{255}=T_{216} R_{15} R_{65}$, where $T_{216}$ is the high order factor of quadrupole, $R_{15}$ and $R_{65}$ are the coupling terms accumulated upstream. It is necessary to add several quadrupoles for correction. In addition, sextupole is also considerable further.

\section{NUMERICAL SIMULATIONS}

To demonstrate the reversible heating system, we employ the beam and LINAC parameters listed in Table I [27] 
TABLE I. The beam and lattice parameters.

\begin{tabular}{lccc}
\hline \hline Parameter & Symbol & Value & Unit \\
\hline Initial energy & $\gamma_{0} m c^{2}$ & 130 & $\mathrm{MeV}$ \\
Energy downstream & $\gamma m c^{2}$ & 250 & $\mathrm{MeV}$ \\
Normalized emittance & $\epsilon_{n}$ & 1 & $\mu \mathrm{m}$ \\
Beam size (rms) & $\sigma_{x_{0}, y_{0}}$ & 300 & $\mu \mathrm{m}$ \\
Beam divergence (rms) & $\sigma_{x_{0}^{\prime}, y_{0}^{\prime}}$ & 13 & $\mu \mathrm{rad}$ \\
Peak current upstream & $I_{0}$ & 50 & $\mathrm{~A}$ \\
Peak current downstream & $I_{f}$ & 500 & $\mathrm{~A}$ \\
Bunch length(FWHM) & $\sigma_{s_{0}}$ & 3 & $\mathrm{~mm}$ \\
Slice energy spread (rms) & $\sigma_{E_{0}}$ & 1.3 & $\mathrm{keV}$ \\
Momentum compaction factor & $R_{64}$ & 56 & $\mathrm{~mm}$ \\
Compression factor & $C$ & 10 & $/$ \\
1st energy chirp & $h_{1}$ & -2 & $\mathrm{~m}^{-1}$ \\
2nd energy chirp & $h_{2}$ & 16 & $\mathrm{~m}^{-1}$ \\
1st TGU strength & $\theta_{1}$ & 0.12 & $/$ \\
1st TGU strength & $\theta_{2}$ & 0.015 & $/$ \\
\hline \hline
\end{tabular}

and track the electron beam through the practical layout as shown in Fig. 1. With the layout and the theoretical analysis in the previous section, we determine appropriate parameters for each optics element following Eq. (6), which are also presented in Table I. With an reference energy of $250 \mathrm{MeV}$, choose energy chirps $h_{1}=-2 \mathrm{~m}^{-1}$ and $h_{2}=16 \mathrm{~m}^{-1}$ to provide a required suppression factor in Eq. (16) and an effective heated energy spread in Eq. (12). TGU strengths of $\theta_{1}=0.12$ and $\theta_{2}=0.015$ are achievable [28,29], for instance, the stronger one (T1) is a 15-period undulator with a 10 -cm period length, the peak magnetic field is $B_{0} \approx 1.8 \mathrm{~T}$ and the transverse gradient is $\alpha=100 \mathrm{~m}^{-1}$.

Under the linear transport approximation, we can give the estimated results based on the theoretical analysis. For the modulation wavelengths of $100 \mu \mathrm{m}$ and $50 \mu \mathrm{m}$, the global suppression factors related to beam size are up to $\sim 0.1$ and $\sim 10^{-4}$, respectively. The slice energy spread is heated from $1.3 \mathrm{keV}$ to $16 \mathrm{keV}$ first and amplified to $160 \mathrm{keV}$ after the bunch compressor, and finally reversed to $13 \mathrm{keV}$ with a compression factor of 10 . The horizontal emittance grows tens of times right after the bunch compressor, and falls back to less than $1.0 \%$ growth at the end. Both the energy spread increase and the emittance growth are almost completely removed except for a residual coupling term at the exit of the system.

For simulation, an initial beam is assumed with Gaussian transverse distributions and flattop longitudinal distribution. We use the particle tracking code ELEGANT [30] to track the beam with 1 million macroparticles through the system, where the TGU is treated as a matrix element referred to Eq. (1).

Nonlinear effects and wakefield effects should be involved in the simulation. The designed parameters are proposed by the linear theory that originally expected the horizontal phase space was independent on the longitudinal position. Due to the nonlinear effects and the collective effects concerns, both the compression factor and the horizontal emittance will be deteriorated. In terms of the projected emittance, the simulated result increases by several tens of times. Therefore, we add several quadrupoles and a sextupole as mentioned above and improve the designed parameters for the overall optimization.

Figure 2 presents the simulation results of the evolution of the longitudinal phase space along the beam line in our reversible heating system. The initial beam is assumed to be with a slice energy spread of $1.3 \mathrm{keV}$. Passing through the first TGU associated with the upstream linac, the slice energy spread is heated directly and up to $\sim 19 \mathrm{keV}$ upstream of the bunch compressor. Afterwards it increases up to $\sim 150 \mathrm{keV}$ through the bunch compressor. Finally, the slice energy spread is reversed by the second TGU and cooled down to $\sim 15 \mathrm{keV}$ with a compression factor of $\sim 10$.

In order to verify the suppression of both the initial modulation driven and the internal collective effects driven microbunching instabilities, an energy modulation with peak amplitude of $3 \mathrm{keV}$ and wavelength of $50 \mu \mathrm{m}$ and a density modulation with peak amplitude of $5 \%$ and wavelength of $50 \mu \mathrm{m}$ are imposed on the initial beam profile, respectively. As shown in Fig. 3, the initial distributions of the energy modulation case and the density modulation case are presented.

During the transport, both the density modulation and energy modulation are smeared out. Figure 4 shows the longitudinal phase space upstream of the bunch compressor. The smearing of the energy distribution is due to the energy spread heating and the smearing of the density

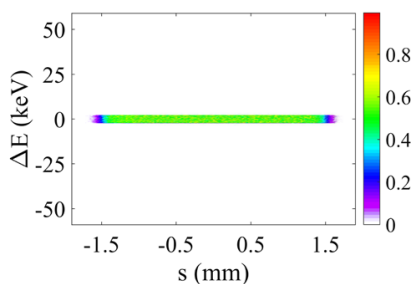

(a) Entrance of the system

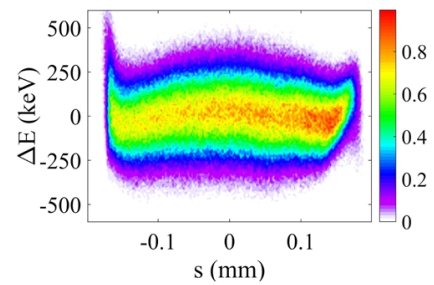

(c) Downstream of BC

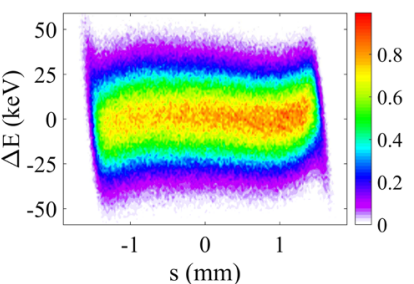

(b) Upstream of BC
FIG. 2. Longitudinal phase space (slice energy spread) with energy chirp removed: (a) at the entrance of the reversible system, upstream of the first linac, (b) upstream of the bunch compressor, (c) downstream of the bunch compressor, upstream of the second TGU, (d) at the exit of the system, downstream of the second TGU. Energy chirp is removed up to the second order, and the bunch head is on the right-hand side. 


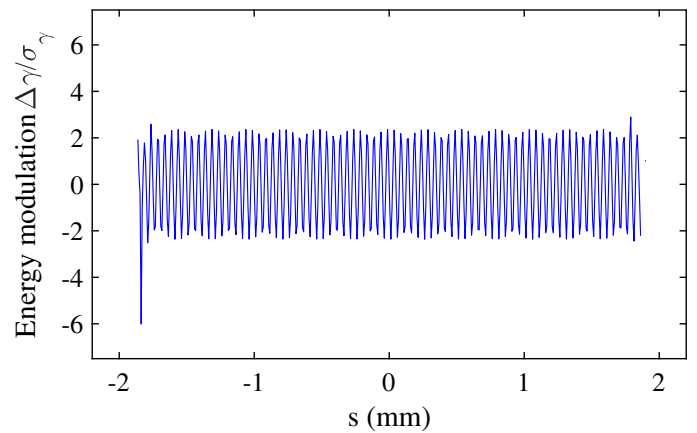

(a) Initial energy modulation case

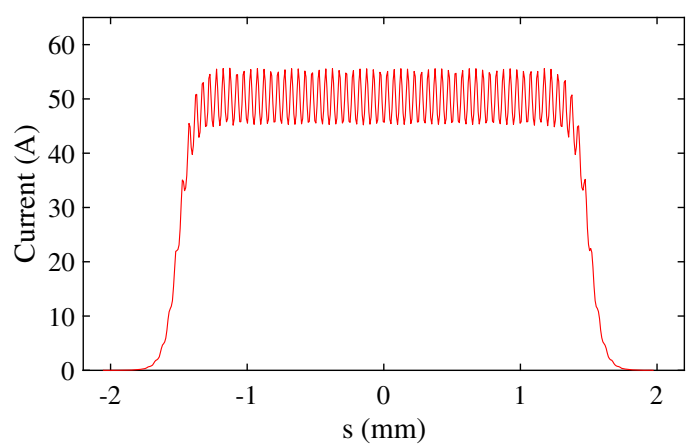

(b) Initial density modulation case

FIG. 3. The initial distributions: (a) the energy modulation amplitude for the case of the initial energy modulation, (b) the current profile for the case of the initial density modulation.

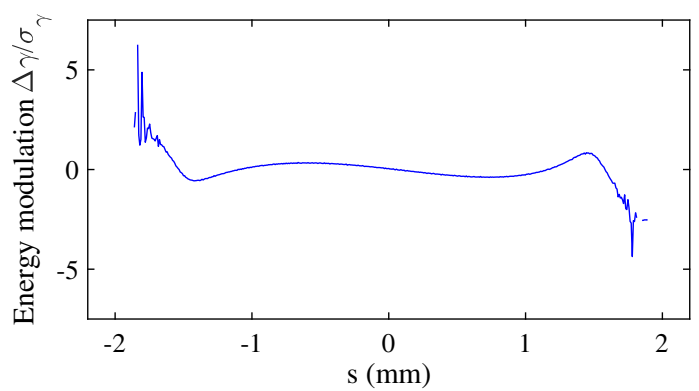

(a) Energy modulation case

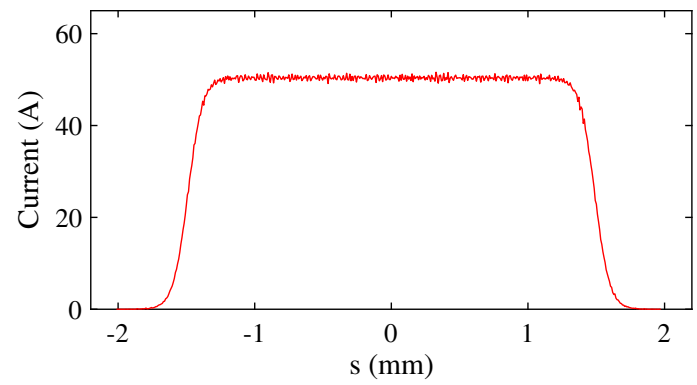

(b) Density modulation case

FIG. 4. Smearing out the density modulation and energy modulation due to the heated energy spread and transverse-tolongitudinal coupling upstream of the bunch compressor. Energy chirp in Fig. 4(a) is removed up the 2nd order. distribution is due to the $s-x$ coupling. These results suppress the accumulation of the internal collective effects on microbunching. Figure 5 describes the centroid of the horizontal distribution with respect to the longitudinal position, which indicates the contribution of the bunch length to horizontal phase space. The coupling is removed by the second TGU (red), which means the horizontal emittance is almost preserved. Simulation results show that the core horizontal emittance growth with $90 \%$ particles is finally less than 5 percent.

To show the suppression of the microbunching instability, we give the results of the energy modulation case and the density modulation case, respectively. For comparison, two cases switching off the TGUs are also simulated. As shown in Fig. 6, without suppression of the TGUs, these two modulation cases present the visible compressed modulation with wavelength of $\sim 5 \mu \mathrm{m}$, i.e., the microbunching is amplified by both LSC and CSR. While switching on the TGUs, the current profile and the longitudinal phase space become smooth and the microbunching instabilities are almost invisible. Compared to the density modulation case, the energy modulation case presents a relatively larger slice energy spread due to the contribution of the initial energy modulation.

As simulated above, both LSC and CSR driven microbunching instabilities accumulated before and inside the reversible heating system would be suppressed. Meanwhile, the heated energy spread is reversed to the initial by the scale of the compression factor, and the diluted horizontal emittance is restored by a few percent growth. Although there is still an emittance growth due to

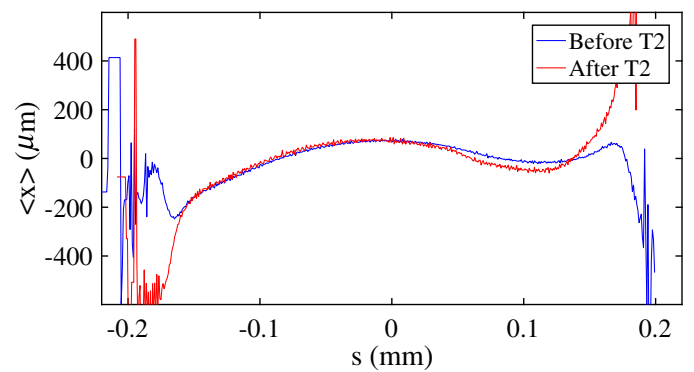

(a) The horizontal position

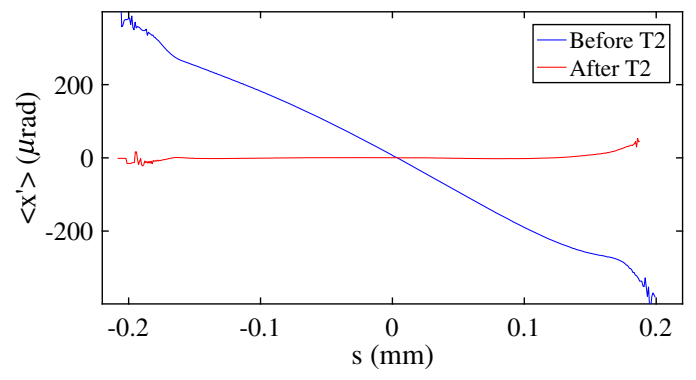

(b) The horizontal momentum

FIG. 5. The centroid of the horizontal distribution with respect to the longitudinal position before and after the second TGU. 


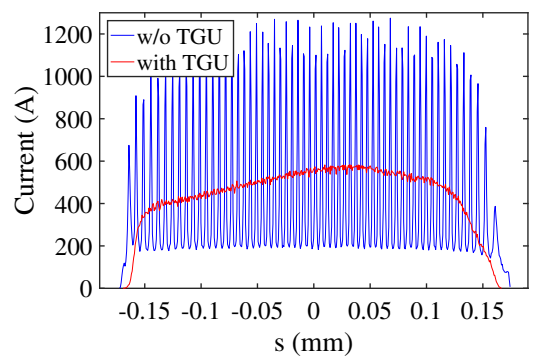

(a) Current for density modulation case

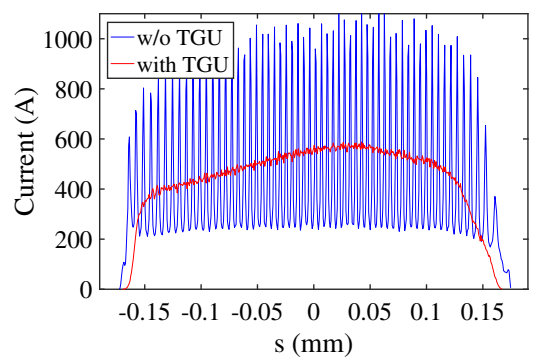

(d) Current for energy modulation case

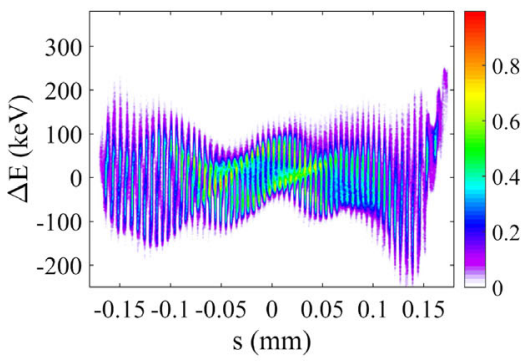

(b) Density modulation case without TGU

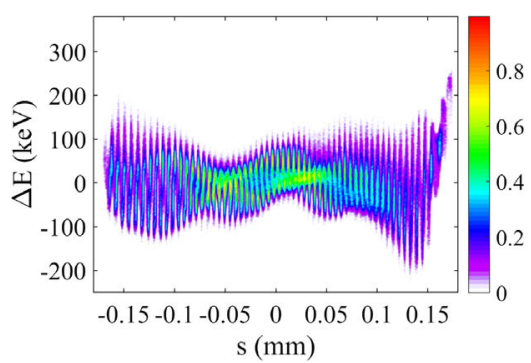

(e) Energy modulation case without TGU

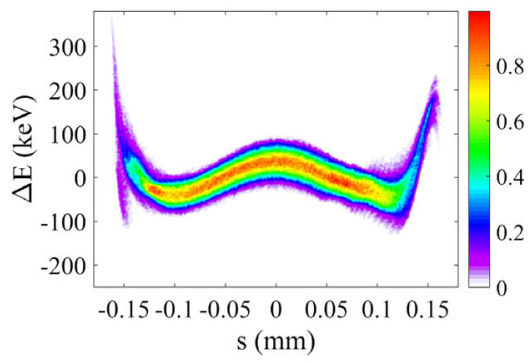

(c) Density modulation case with TGU

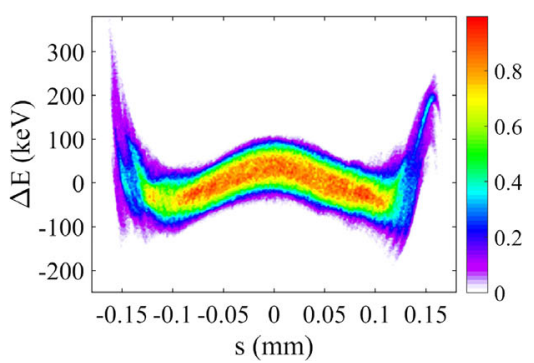

(f) Energy modulation case with TGU

FIG. 6. Suppression of the microbunching instabilities due to the initial density modulation and the initial energy modulation, i.e., simulating the initial collective effects driven modulation and the internal collective effects driven modulation. Current profiles (a, d) and longitudinal phase space (b, c, e, f) are presented. Energy chirps up to the 2nd order are removed.

the residual $R_{26}$ noted in the matrix (8), the coupling is dominated by the initially uncorrelated energy spread rather than the global energy spread of the chirped beam. Therefore the leaked dispersion would not considerably affect the beam transport downstream.

\section{PRACTICAL EFFECTS}

Some practical issues need to be considered in the system. In the previous sections, we have discussed the principle of the microbunching instability suppression and the beam heating reversion by means of the theoretical analysis, calculations and numerical simulations. In the simulations, the nonlinear transport and wakefields are also included in elegant code, except for those of the TGUs. Accordingly, we calculate the dominant high order terms related to the longitudinal position such as $T_{215}$ and $T_{255}$ for the TGUs, which are also able to be canceled by adjusting the quadrupoles and the harmonic cavity. As a result, the core emittance is still only increased by a few percent. For a practical TGU, more high order terms like horizontal-tovertical coupling and wakefields are inevitable which are neglected in our studies.

We also discuss the impact of rf jitter on the system, which plays an important role in the jitter analysis. Using the rf phase jitter as an example, a jitter of $0.1 \mathrm{deg}$ rms can cause both central energy and time-energy chirp jitter. Under the condition in Eq. (7), the case described in Eq. (8) is not exactly fulfilled, i.e., the system does not reverse the electron beam exactly resulting in a horizontal emittance growth and a slice energy spread increase. As shown in Fig. 7, the phase jitter leads to the changes of the compression factor and the reversed slice energy spread to a tolerable level, and the core horizontal emittance is also increased by less than 10 percent. Besides, the energy jitter

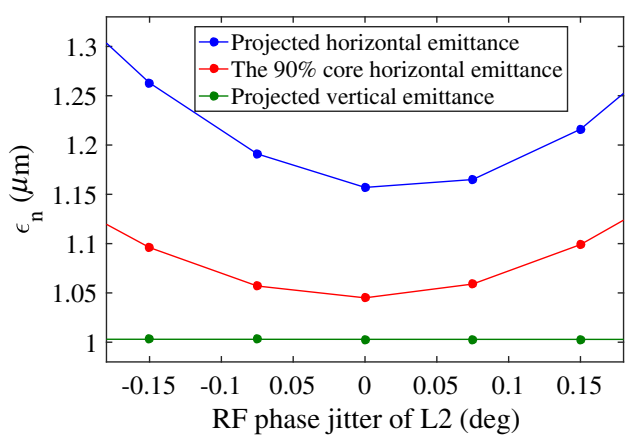

(a) Transverse phase space

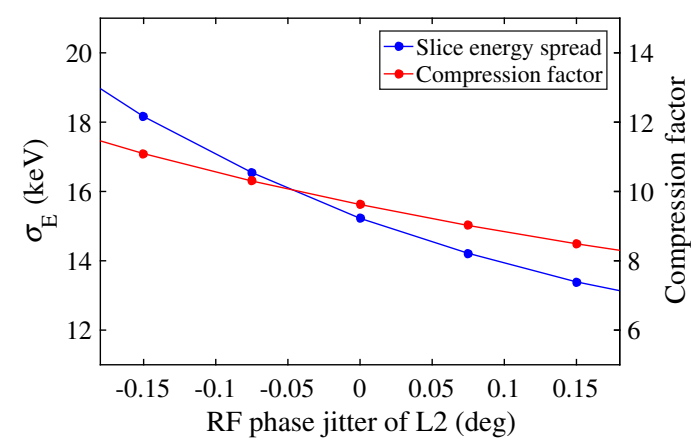

(b) Longitudinal phase space

FIG. 7. The effects of the rf phase jitter on the electron beam in the system. (a) Normalized projected emittances in transverse phase space, (b) slice energy spread and compression factor in longitudinal phase space. 
due to voltage and phase jitter in the linac may lead to a jitter of arrival time. Considering the $R_{56}$ term of the TGU, we can derive the overall $R_{56}$ term which is given by

$$
R_{56}^{\text {total }}=R_{56}^{A}+\left(1+h_{2} R_{56}^{A}\right)\left(R_{56}+R_{56}^{B}\right)
$$

with the terms $R_{56}^{A}$ and $R_{56}^{B}$ of TGU1 and TGU2 referred in Eq. (1). In our system, the total $R_{56}$ change induced by TGUs is on the order of $10^{-3}$, which is less than 10 percent compared to the case without TGUs. Therefore the arrival time jitter is mainly introduced by the bunch compressor.

In our case, the transverse dispersion introduced by TGU dominates the tolerance in the horizontal dimension. The first TGU generates a dispersion with $R_{26}=0.1$ that will be accumulated to a maximum rms horizontal beam size of $\sim 1 \mathrm{~mm}$ close to the entrance of the bunch compressor. In addition, the time dependent kick and focusing in the linac induce an off-axis of the beam due to the transverse wake on a tilted beam, therefore several correctors are required in this system.

\section{SUMMARY}

In this paper we have proposed a reversible electron beam heating system based on transverse gradient undulators, that can suppress the microbunching instability and preserve the high beam quality at the same time. Theoretical analysis and numerical simulations show that there are two factors generated in the system to suppress the microbunching instability, i.e., the heated energy spread and the horizontal-to-longitudinal coupling. These two factors can smear out the modulated energy distribution and the accumulated density distribution. The heated energy spread performs an action on suppression of the microbunching instability driven by LSC and CSR within the system. The horizontal-to-longitudinal coupling not only suppresses the source of LSC and CSR effects, but also suppresses the microbunching instability generated by the initial modulation thanks to a residual coupling at the end of the system. As a result, the microbunching originated from the initial energy and density modulation are smeared out, which means both the initial and internal collective effects including LSC and CSR driven microbunching instabilities are suppressed eventually. The slice energy spread is restored to the initial uncorrelated energy spread enlarged by a scale factor of the compression factor. The transverse emittance growth is almost reversed although with a slight degradation due to the nonlinear effects and the collective effects, typically the core emittance is preserved very well. Other than a dipole, a TGU does not deflect the beam line downstream as a potential advantage of the beam transport. Consequently, the reversible heater based on TGUs enables the application to the suppression of the microbunching instability in a linear accelerator and indicates the feasibility to improve the seeded FEL performance.

\section{ACKNOWLEDGMENTS}

We would like to thank G. Stupakov, K.-J. Kim, Y. Ding, P. Baxevanis, Y. Cai, C. Feng, and D. Huang for useful discussions and suggestions. This work was partially supported by the U.S. National Science Foundation (Grant No. PHY-1535215), the U.S. Department of Energy Contract No. DE-AC02-76SF00515, and the Ministry of Science and Technology of China (Grants No. 2016YFA0401900).

[1] C. Bostedt, S. Boutet, D. M. Fritz, Z. Huang, H. Ja Lee, H. T. Lemke, A. Robert, W. F. Schlotter, J. J. Turner, and G. J. Williams, Linac coherent light source: The first five years, Rev. Mod. Phys. 88, 015007 (2016).

[2] W. Ackermann et al., Operation of a free-electron laser from the extreme ultraviolet to the water window, Nat. Photonics 1, 336 (2007).

[3] P. Emma et al., First lasing and operation of an angstromwavelength free-electron laser, Nat. Photonics 4, 641 (2010).

[4] T. Ishikawa et al., A compact x-ray free-electron laser emitting in the sub-angstrom region, Nat. Photonics 6, 540 (2012).

[5] E. Allaria et al., Two-stage seeded soft-x-ray free-electron laser, Nat. Photonics 7, 913 (2013).

[6] E. L. Saldin, E. A. Schneidmiller, and M. V. Yurkov, Longitudinal space charge-driven microbunching instability in the tesla test facility linac, Nucl. Instrum. Methods Phys. Res., Sect. A 528, 355 (2004).

[7] E. L. Saldin, E. A. Schneidmiller, and M. V. Yurkov, On the coherent radiation of an electron bunch moving in an arc of a circle, Nucl. Instrum. Methods Phys. Res., Sect. A 398, 373 (1997).

[8] M. Borland et al., Start-to-end simulation of self-amplified spontaneous emission free electron lasers from the gun through the undulator, Nucl. Instrum. Methods Phys. Res., Sect. A 483, 268 (2002).

[9] Z. Huang and K.-J. Kim, Formulas for coherent synchrotron radiation microbunching in a bunch compressor chicane, Phys. Rev. ST Accel. Beams 5, 074401 (2002).

[10] E. L. Saldin, E. A. Schneidmiller, and M. V. Yurkov, Klystron instability of a relativistic electron beam in a bunch compressor, Nucl. Instrum. Methods Phys. Res., Sect. A 490, 1 (2002).

[11] S. Heifets, G. Stupakov, and S. Krinsky, Coherent synchrotron radiation instability in a bunch compressor, Phys. Rev. ST Accel. Beams 5, 064401 (2002).

[12] Z. Huang, M. Borland, P. Emma, J. Wu, C. Limborg, G. Stupakov, and J. Welch, Suppression of microbunching instability in the linac coherent light source, Phys. Rev. ST Accel. Beams 7, 074401 (2004).

[13] Z. Huang et al., Measurements of the linac coherent light source laser heater and its impact on the x-ray free-electron laser performance, Phys. Rev. ST Accel. Beams 13, 020703 (2010). 
[14] S. Spampinati et al., Laser heater commissioning at an externally seeded free-electron laser, Phys. Rev. ST Accel. Beams 17, 120705 (2014).

[15] L. H. Yu, Generation of intense uv radiation by subharmonically seeded single-pass free-electron lasers, Phys. Rev. A 44, 5178 (1991).

[16] C. Behrens, Z. Huang, and D. Xiang, Reversible electron beam heating for suppression of microbunching instabilities at free-electron lasers, Phys. Rev. ST Accel. Beams 15, 022802 (2012).

[17] J. Qiang, Chad E. Mitchell, and M. Venturini, Suppression of Microbunching Instability Using Bending Magnets in Free-Electron-Laser Linacs, Phys. Rev. Lett. 111, 054801 (2013).

[18] C. Feng, D. Huang, H. Deng, Q. Gu, and Z. T. Zhao, Suppression of microbunching instability via a transverse gradient undulator, New J. Phys. 17, 073028 (2015).

[19] D. Huang, C. Feng, H. Deng, Q. Gu, and Z. T. Zhao, Transverse to longitudinal phase space coupling in an electron beam for suppression of microbunching instability, Phys. Rev. Accel. Beams 19, 100701 (2016).

[20] G. Stupakov and P. Emma, in Proc. 37th International Free Electron Laser Conference (FEL2015), Daejeon, Korea (JACoW, Geneva, Switzerland, 2015), p. 166.

[21] T. I. Smith, J. M. J. Madey, L. R. Elias, and D. A. G. Deacon, Reducing the sensitivity of a free-electron laser to electron energy, J. Appl. Phys. 50, 4580 (1979).

[22] Z. Huang, Y. Ding, and Carl B. Schroeder, Compact X-ray Free-Electron Laser from a Laser-Plasma Accelerator
Using a Transverse-Gradient Undulator, Phys. Rev. Lett. 109, 204801 (2012).

[23] T. Liu, T. Zhang, D. Wang, and Z. Huang, Compact beam transport system for free-electron lasers driven by a laser plasma accelerator, Phys. Rev. Accel. Beams 20, 020701 (2017).

[24] P. Baxevanis, Y. Ding, Z. Huang, and R. Ruth, 3d theory of a high-gain free-electron laser based on a transverse gradient undulator, Phys. Rev. ST Accel. Beams 17, 020701 (2014).

[25] T. Liu, W. Qin, Y. Ding, D. Wang, and Z. Huang, in Proceedings of IPAC2017 (JACoW, Geneva, Switzerland, 2017).

[26] K. L. Brown, Technical Report No. SLAC-75, 1982.

[27] Z. T. Zhao, S. Y. Chen, L. H. Yu, C. X. Tang, L. X. Yin, D. Wang, and Q. Gu, in Proceedings of the 2nd International Particle Accelerator Conference, San Sebastiáán, Spain (EPS-AG, Spain, 2011).

[28] V. A. Rodríguez, A. Bernhard, A. Keilmann, P. Peiffer, R. Rossmanith, C. Widmann, T. Baumbach, M. Nicolai, and M.C. Kaluza, Development of a superconducting transverse-gradient undulator for laser-wakefield accelerators, IEEE Trans. Appl. Supercond. 23, 4101505 (2013).

[29] A. Bernhard, N. Braun, V. A. Rodríguez, P. Peiffer, R. Rossmanith, C. Widmann, and M. Scheer, Radiation emitted by transverse-gradient undulators, Phys. Rev. Accel. Beams 19, 090704 (2016).

[30] M. Borland, Argonne National Laboratory Advanced Photon Source Report No. LS-287, 2000. 